\title{
VENTRICULAR ESCAPE WITH OBSERVATIONS ON CASES SHOWING A VENTRICULAR RATE GREATER THAN THAT OF THE, AURICLES *
}

\author{
PAUL D. WHITE, M.D. \\ BOSTON
}

The ventricles occasionally escape from the control of the sinuauricular node through the independent action of their own center of stimulus production in the atrioventricular junctional tissues. Two types of such ventricular escape should be recognized: first, that which is dependent primarily on a depression of the pacemaker in the sinuauricular node; and second, that which is dependent primarily on an excitation of the pacemaker in the atrioventricular node. As one would 1 anticipate, examples of the first type are occasionally seen, while instances of the second type appear to be decidedly rare, though both conditions may be more common than we realize at present. The two factors, depression of the sinu-auricular node and the excitation of the atrioventricular node, may be found in a single case. The differentiation between the two types must be based largely on the rate of the sinu-auricular pacemaker from which the ventricles escape and upon the rate of the ventricular pacemaker which escapes. In the electrocardiogram the escaped ventricular complex is normal in shape, similar to that resulting from auricular excitation.

Sometimes the atrioventricular node may manufacture the stimulus for both auricles and ventricles, thus producing true atrioventricular rhythm. This should be distinguished from simple ventricular escape, for in atrioventricular rhythm only one pacemaker controls the heart, while in escape of the ventricles there are two pacemakers, both nodes functioning, the upper node for the auricles and the lower node for the ventricles. Atropin injected subcutaneously may release the lower node from vagal action before it releases the upper node, so that ventricular escape may occur temporarily, as pointed out by Gallavardin, Dufourt and Petzetakis $;^{1}$ or true atrioventricular rhythm may occur spontaneously after atropin for a few minutes, as pointed out in one case by Wilson ${ }^{2}$ and as seen in a recent case at the Massachusetts General Hospital (Fig. 1). Wilson has produced this rhythm in a number of

* Submitted for publication April 6, 1916.

* From the Medical Service of the Massachusetts General Hospital.

1. Gallavardin, L.; Dufourt, P., and Petzetakis: Arch. d. mal du cour, 1914, vii, 1.

2. Wilson, F. N.: The Archives Int. Med., 1915, xvi, 989. 
subjects by the combined effect of early atropin action and ocular pressure or forced respiration. The differential diagnosis between ventricular escape and atrioventricular rhythm is very uncertain without the electrocardiogram. Errors may easily be made in the interpretation of polygrams, in the jugular records of which the $a$ and $c$ waves almost or completely coincide. Even if the shortened $a-c$ or the $c$ - $a$ intervals are constant for a considerable stretch of record, ventricular escape may be occurring and not atrioventricular rhythm. In the electrocardiogram, however, we have in the shape of the auricular complex an invaluable clue to the true condition. If the shape of this complex is normal, we have evidence immediately that the sinu-auricular node is
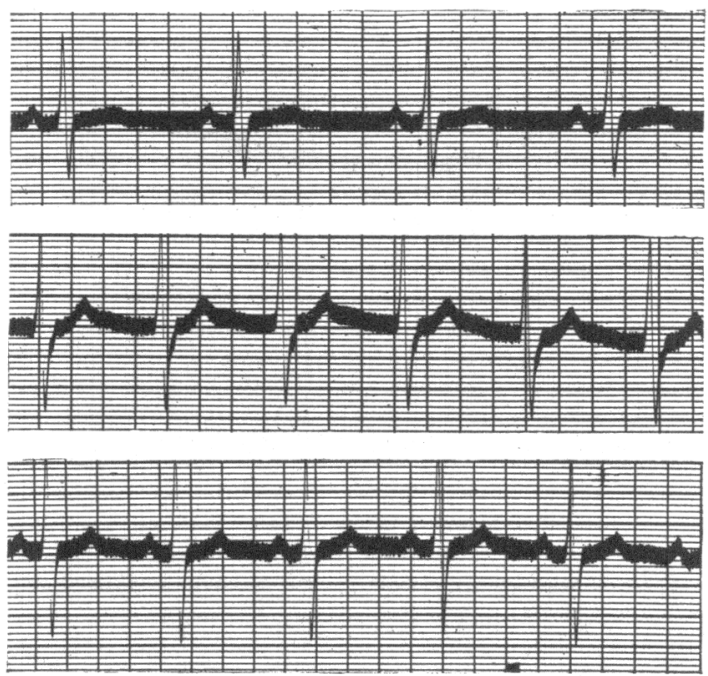

Fig. 1.-Lead 2 of electrocardiogram of E. B. Upper record, before atropin; middle record, fifteen minutes after atropin sulphate $0.002 \mathrm{gm}$. subcutaneously; and lower record, thirty minutes after the injection of atropin. In the upper and lower records normal rhythm arising in the sinu-auricular node is present; in the middle record occurs a transient atrioventricular rhythm, the inverted auricular complex falling at the end of the first ventricular complex. In all figures abscissae $=0.2$ second, ordinates $=10^{-4}$ volts. The curves in this and the following illustrations are reduced to two-thirds size of the originals.

functioning; if the auricular complex is inverted (in Lead 2), and always at the same time interval immediately before or after the first ventricular complex, we may conclude that the atrioventricular node is probably controlling both auricles and ventricles.

The auriculoventricular dissociation found in ventricular escape is different from that found in complete heart block in that there is usually no defect in auriculoventricular conduction with it. In highgrade heart block, however, ventricular escape may occur as the result 
of such a blocking of auricular stimuli that the idioventricular rhythm asserts itself, even though atrioventricular node and bundle may still be able to carry an impulse now and then.

TYPES OF VENTRICLLAR ESCAPE

Belonging to the type of ventricular escape for which depression and slowing of the sinu-auricular pacemaker are primarily responsible, are several conditions. When the vagus nerve is overactive, forced expiration may produce a temporary dissociation of the auricles and ventricles, due to marked slowing in the rate of the auricle with resultant escape of the atrioventricular nodal pacemaker, as seen in

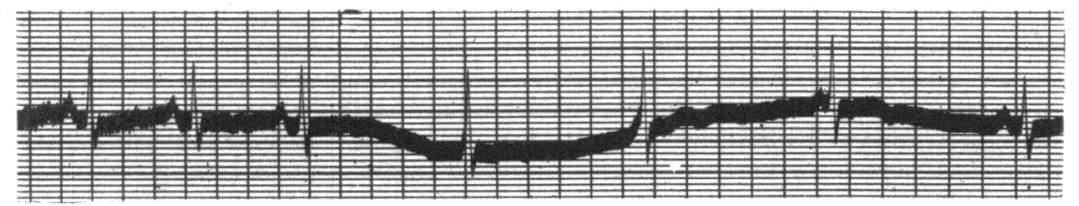

Fig. 2.-Lead 2 of electrocardiogram of G. A. M., showing ventricular escape as the result of forced expiration.
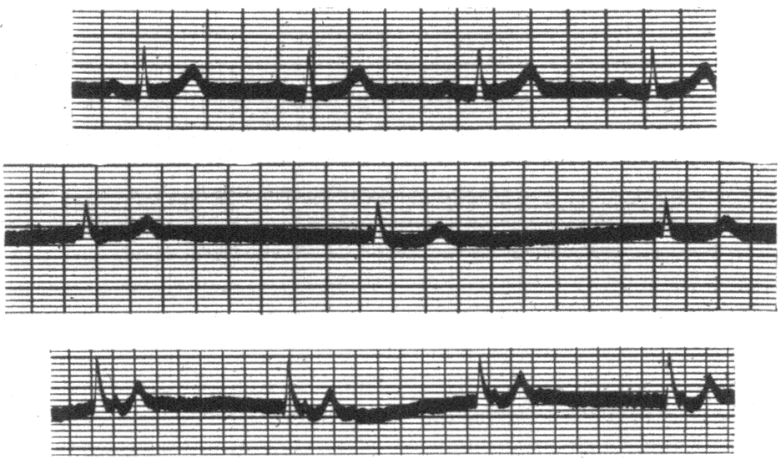

Fig. 3.-Lead 1 of electrocardiogram of G. H. A., showing auriculoventricular dissociation resulting from digitalis. In the upper record occurs the normal rhythm of the patient while not under the influence of digitalis.

ligure 2. Vagal pressure may also act to produce ventricular escape, by depressing the sinu-auricular nodal rate below that of the atrioventricular node (Fig. 6). Digitalis may be responsible for such a situation, as shown by Figure 3. Finally it may occur without obvious cause (Fig. 4). Gallavardin, Dufourt, and Petzetakis ${ }^{1}$ have shown that the most varied figures of auriculoventricular superposition in polygrams and electrocardiograms may be produced by intermittent ventricular automatism, as in three patients with bradycardia who came under their observation. 
An irritable atrioventricular node may show itself by ventricular escape, even when the rate of stimulus production in the sinu-auricular node is not low. In such cases the ventricular rate may be higher than the auricular rate. Recently at the Massachusetts General Hospital $I$ have seen the ventricular rate spontaneously increased over that of the auricles in a woman 24 years of age who had suffered four weeks before from a peritonsillar abscess and who had had a tonsillectomy under ether four days before the cardiac peculiarity was discovered. She had had no drugs. After her tonsillectomy she had noticed slight but distinct palpitation, which she had never experienced before. Her rate was higher than usual, 90 to 100 instead of about 70 . There was occasionally slight quickening of her pulse on palpation; until I obtained graphic records, I thought this slight arrhythmia might be due to auricular premature beats. An electrocardiogram showed auriculoventricular dissociation, with a ventricular rate of 96 and an auricular rate ranging from 67 to 85 (Fig. 5). The interventricular
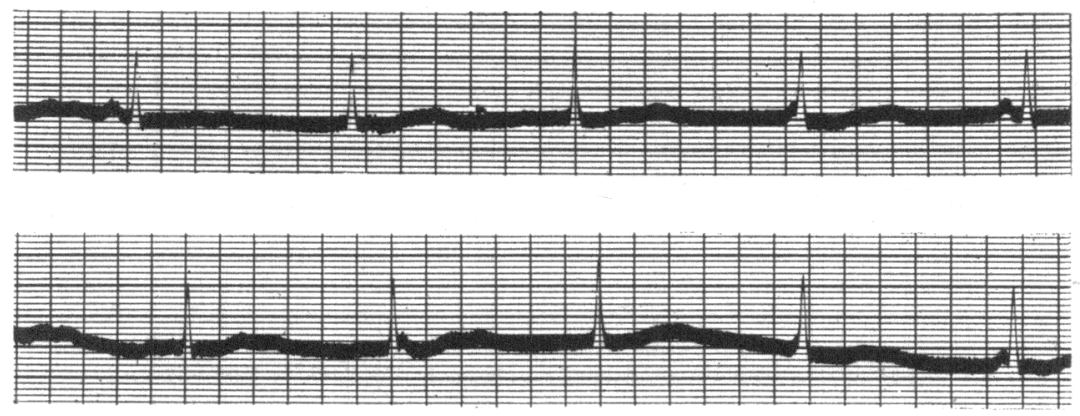

Fig. 4.-Lead 2 of electrocardiogram of A. P., showing auriculoventricular dissociation.

interval of 0.66 second was very constant, except when the auricular excitation came just after the ventricle had recovered from its refractory period and before enough time had elapsed for its escape. At such times the $P$ deflection in the electrocardiogram is seen to fall on the $T$ wave of the ventricular complex. A shortening of the interventricular interval $R$ to $R$ results, thus interrupting momentarily the ventricular dominant rhythm in the node of Tawara. In this patient interesting additional evidence was obtained as the result of her resumption of normal rhythm, at a rate of 78 to 83 , the day after the dissociation was discovered, and the temporary return of the dissociation as the result of either right or left vagal pressure (Fig. 6). On this day the slowing of the pacemaker in the sinu-auricular node from 80 to 68 allowed the escape of the pacemaker in the atrioventricular node. Here apparently was an irritable atrioventricular node, less irritable on the 
second day of examination than on the first, but still irritable enough to escape when the sinu-auricular rate was somewhat slowed. The immediate cause of this irritability appears to have been the tonsillectomy, but the modus operandi is a matter of conjecture at present. Aside from the dissociation in this case the heart appeared normal. The patient felt well and was up and about. Wilson ${ }^{3}$ in 1915 reported a case in which, as the result of forced respiration, a dissociation of auricles and ventricles occurred with a ventricular rate of about 85 and an auricular rate of about 75 . This was found in a man of 22 years.

An increase of the rate of the ventricles beyond that of the auricles is uncommon. Lea ${ }^{4}$ in 1915 collected two cases from the literature and reported one himself in which the ventricular rate exceeded that of the auricles in complete heart block. Of these three cases, digitalis

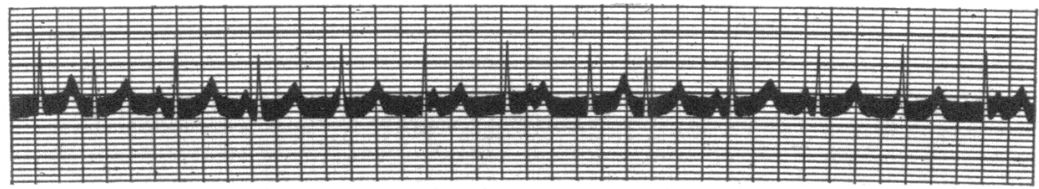

Fig. 5.-Lead 2 of electrocardiogram of J. R. O., showing spontaneous ventricular escape, with higher ventricular than auricular rate.

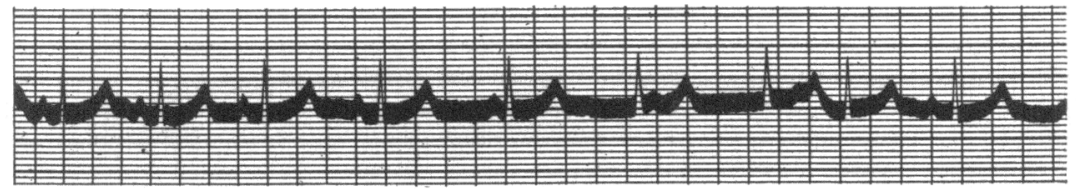

Fig. 6.-Lead 2 of electrocardiogram of J. R. O., showing transient ventricular escape resulting from pressure of left vagus nerve.

was apparently responsible for one (Meyer's) and strophanthus for another (Hewlett and Barringer's) ; Lea's patient had had no digitalis. A rate of the ventricles higher than that of the auricles occurred temporarily in a case of Gallavardin's ${ }^{1}$ and in one of Wilson's ${ }^{2}$ due to ventricular escape resulting from the administration of atropin. Forced respiration resulting in ventricular escape increased the rate of the ventricles over that of the auricles in the case of Wilson, ${ }^{3}$ to which reference has already been made.

Three cases have come under my observation at the Massachusetts General Hospital which showed increase in the rate of the ventricles over that of the auricles. One, occurring spontaneously, has been described above; the other two can be directly ascribed to digitalis,

3. Wilson, F. N.: The Archives Int. Med., 1915, xvi, 86.

4. Lea, Edgar: Lancet, London, 1915, i, 1289. 
in one the automatic ventricular rate varying from 65 to 82 while the auricular rate was constant at 41 to 43 (Fig. 7). In the first curve of Figure 7 a regular ventricular action of 82 to the minute occurred, every other beat apparently responding to a regular auricular action of 41 to the minute. In the lower record the shorter interventricular intervals which disturb the dominant rhythm of the ventricles are produced by transient auriculoventricular association, with prolonged $P-R$. interval, as the result of the falling of the auricular deflection beyond the refractory period of the ventricle, but not late enough to allow the ventricular pacemaker to escape. In this case both factors in the production of ventricular escape are present. The other case in which digi-
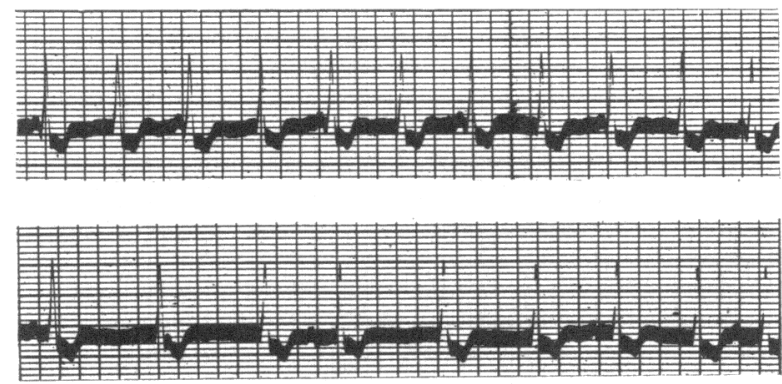

Fig. 7.--Lead 2 of electrocardiogram of E. J. Y., showing ventricular escape resulting from digitalis. In the upper record the ventricular rate is 82 and the auricular rate 41 ; in the lower record the ventricular rate is 65 and the auricular rate 43 .

talis was responsible for the higher ventricular rate, was a patient with atrioventricular rhythm, in whom a curious bigeminy occurred after digitalis. This bigeminy consisted of the sandwiching of an auricular beat between two ventricular contractions; a full description of it may be found in a previous paper by the writer.

\section{SUMMARY}

Two types of ventricular escape are described: (1) the occasional type, in which the automatic stimulus production in the atrioventricular node is released by depression, and hence slowing, of the pacemaker in the sinu-auricular node; and (2) the rare type, in which the atrioventricular nodal center of stimulus production is so irritable that it escapes from the control of the sinu-auricular node. Both factors, depression of the upper node and irritation of the lower node, may play a part in ventricular escape in a single case, as in an instance reported, in the production of which digitalis apparently was the important factor. Three cases are recorded in which the ventricular rate exceeded that of the auricles.

5. White, P. D.: The Archives Int. Med., 1915, xvi, 517. 